\title{
Endovascular treatment of erectile dysfunction due to internal iliac artery atherosclerotic disease
}

\author{
Bryan P Yan *, MB, BS, FRACP \\ Department of Medicine and Therapeutics, The Chinese University of Hong Kong, Shatin, Hong Kong \\ * Corresponding author: bryan.yan@cuhk.edu.hk
}

Hong Kong Med J 2018;24:629-31

DOI: $10.12809 / \mathrm{hkmj} 154810$

\section{Introduction}

Erectile dysfunction (ED) is defined as the recurrent inability to achieve and maintain an erection satisfactory for sexual intercourse. Worldwide, ED affects approximately 300 million men, and nearly $30 \%$ of men between age 40 and 70 years. ${ }^{1}$ Current pharmacological options for treatment of ED are limited, with up to $50 \%$ of patients experiencing suboptimal response to oral phosphodiesterase inhibitor (PDE5i). ${ }^{2}$ Atherosclerotic occlusive disease of the ilio-pudendal-penile arteries resulting in arterial insufficiency to the penis has been reported to affect up to $75 \%$ of patients with ED. ${ }^{3}$ In a recent study of 30 diabetic patients with ED and coronary artery disease, significant internal iliac artery (IIA) and internal pudendal artery (IPA) disease was found $20 \%$ and $36.7 \%$ of patients, respectively. ${ }^{4}$ Atherosclerotic occlusive disease may result in arterial insufficiency to the penis, limiting the inflow of blood required to fill the corpora cavernosa to achieve penile erection. This report describes the case of a patient with ED caused by atherosclerotic disease of the left IIA that was successfully treated by endovascular revascularisation.

\section{Case presentation}

A 71-year-old man presented to our hospital with recurrent claudication and severe erectile dysfunction (ED). The patient had a history of insulinrequiring type 2 diabetes mellitus, hypertension, hyperlipidaemia, renal transplant for end-stage renal failure due to obstructive nephropathy, advanced coronary and peripheral artery disease status after multiple interventions. The patient had been diagnosed with ED more than 10 years previously. His ED responded initially to oral phosphodiesterase inhibitor (PDE5i) therapy. In the 12 months prior to the present case, the patient's ED deteriorated and became refractory to PDE5i therapy. This caused significant distress and loss of quality of life because the patient wished to remain sexually active.

Peripheral angiography of the ilio-pudendalpenile arteries was performed opportunistically at time of endovascular intervention for recurrent lower limb claudication in September 2015. The peripheral angiography revealed total occlusion of the right IIA and haemodynamically significant stenoses in the left common and distal IIA with a significant pressure gradient of $20 \mathrm{~mm} \mathrm{Hg}$ across the lesions (Fig 1).

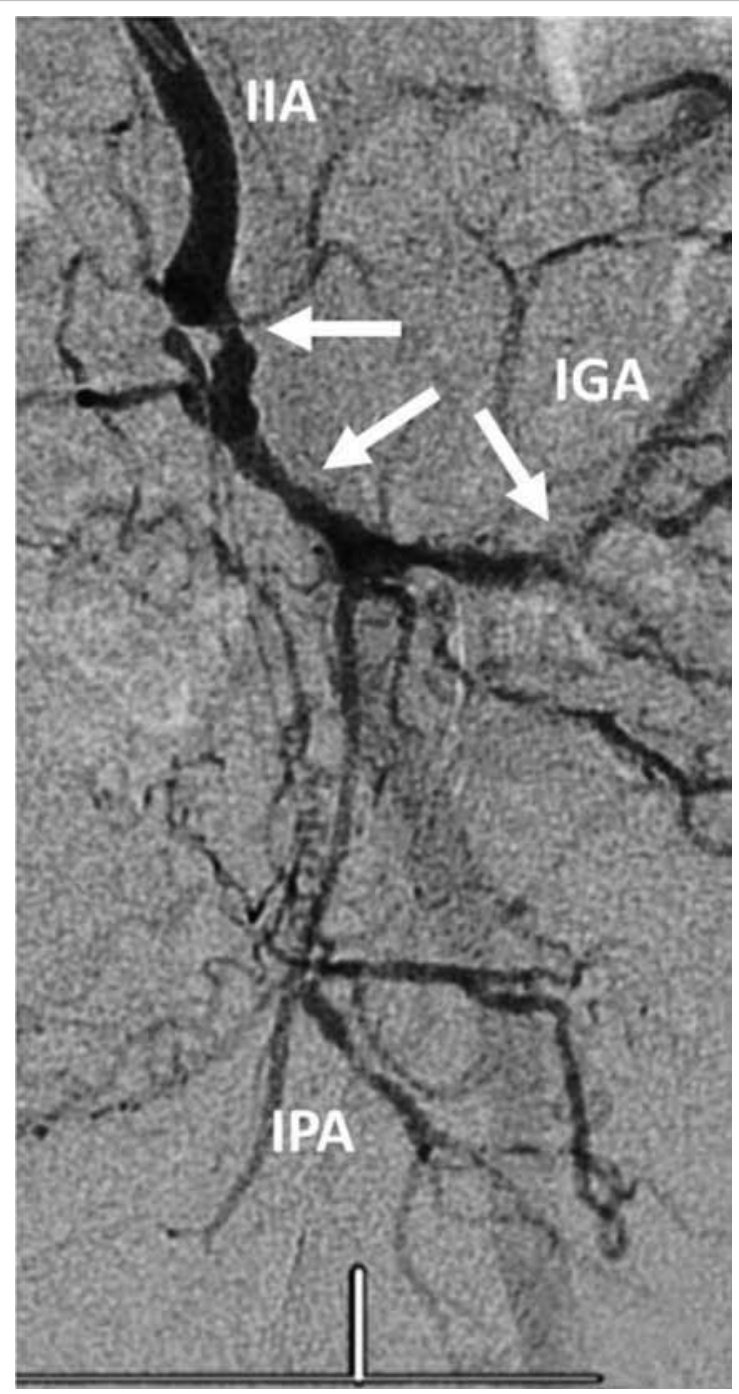

FIG I. Angiography of the left ilio-pudendal-penile arterial system showing significant stenoses (white arrows) in the distal internal iliac artery (IIA) with a translesional gradient of $20 \mathrm{~mm} \mathrm{Hg}$. This resulted in poor distal flow into the internal pudendal artery (IPA) and inferior gluteal artery (IGA) 
The penile artery and left IPA were supplied by the diseased left IIA. Elective endovascular intervention was performed via a $45-\mathrm{cm}$ 6-Fr sheath inserted in the right common femoral artery and advanced over the aortic bifurcation to the left IIA. The IIA stenosis was dilated with a 4- $\times 40-\mathrm{mm}$ balloon (Sterling; Boston Scientific, Marlborough [MA], United States) followed by a 5- $\times 40$-mm paclitaxel-eluting balloon (Ranger; Boston Scientific) aiming to lower the risk of restenosis. The ostium of the left internal IIA was dilated with a $6-\times 40-\mathrm{mm}$ scoring balloon (Angiosculpt; AngioScore, Fremont [CA], United States) and the left common IIA was dilated with an 8- $\times 20-\mathrm{mm}$ balloon (Sterling; Boston Scientific). Excellent angiographic results were achieved with elimination of the translesional gradient (Fig 2). There were no complications, and the patient was discharged the following day.

The patient reported significant improvement in erectile function at the 3-month follow-up visit. His erectile hardness score improved from 1 (the penis is larger than normal but not hard) to 3 (the penis is hard enough for penetration but not completely hard). Of the domains assessed by the international index of erectile function score: (i) erectile function improved from severe (score 1 out of 30) to moderate dysfunction (score 9); (ii) orgasmic function from severe (score 0 out of 10) to mild-to-moderate dysfunction (score 6); (iii) intercourse satisfaction from severe (score 0 out of 15) to moderate dysfunction (score 4), and (iv) overall satisfaction from moderate (score 4 out of 10) to mild dysfunction (score 8).

\section{Discussion}

With the advancement of endovascular techniques and technologies, revascularisation of the smallcalibre penile artery (average 1-2 $\mathrm{mm}$ ) and IPA (2-3 $\mathrm{mm})$ has been shown to be safe, feasible, and associated with significant improvement in erectile function in selected ED patients with penile arterial insufficiency. ${ }^{5,6}$ In a study of 30 patients with ED and suboptimal response to PDE5i, percutaneous revascularisation of the IPA using coronary drug-eluting stents was associated with clinically meaningful improvement in ED. ${ }^{5}$ Another recently published study on pelvic revascularisation investigated 20 patients with arteriogenic ED. ${ }^{6}$ After balloon angioplasty for isolated penile artery stenosis, $60 \%$ of those patients demonstrated significant improvement in ED at 6 months. ${ }^{6}$ Drug-eluting balloons have been shown to be superior to plain balloon angioplasty in the treatment of infrainguinal peripheral arterial disease. However, the use of drugeluting balloons in the IIA is an off-label indication and should be considered investigational.

In summary, endovascular treatment of iliopudendal-penile arterial disease may offer significant

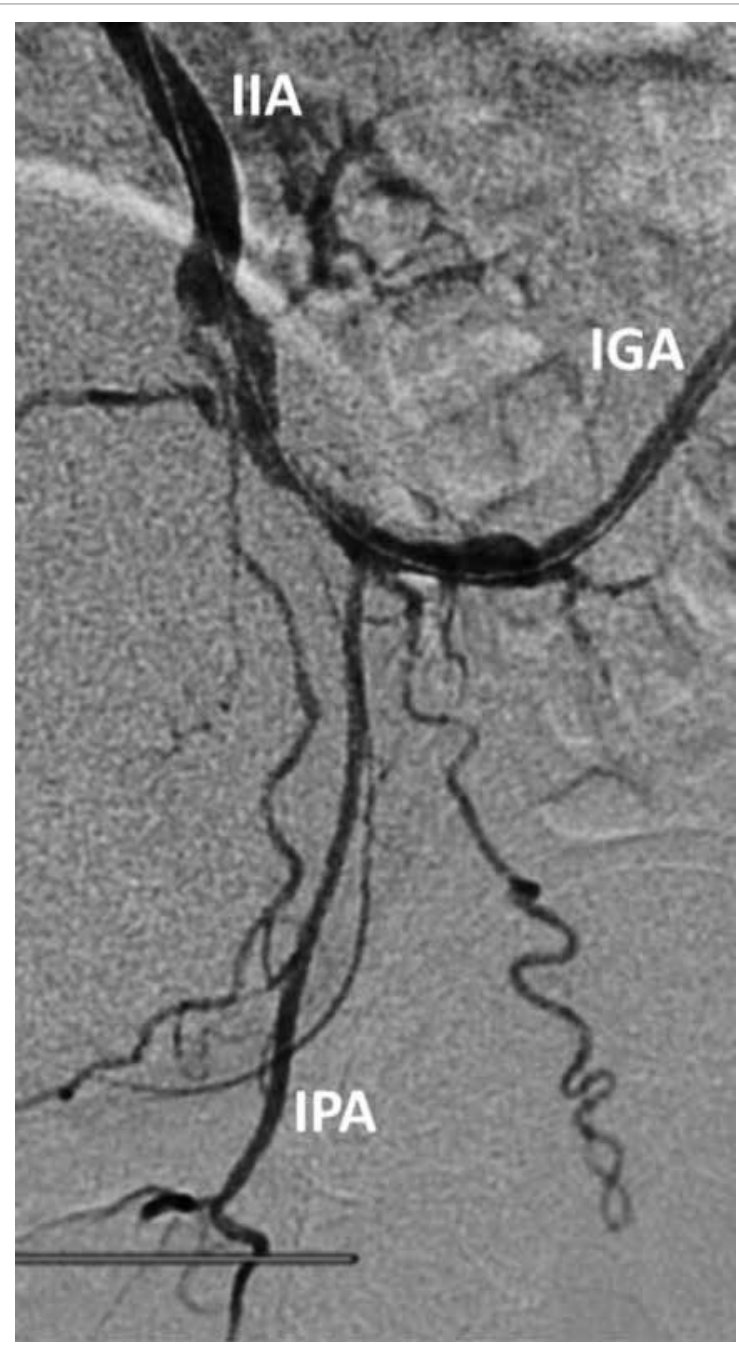

FIG 2. Excellent angiographic results post-angioplasty of the left internal iliac artery (IIA) using a drug-eluting balloon with resolution of translesional gradient. This resulted in improved distal flow into the internal pudendal artery (IPA) and inferior gluteal artery (IGA)

benefits in patients with ED associated with arterial insufficiency that is refractory to medical therapy. However, it is important to emphasise that there are many causes of ED and atherosclerotic disease of erectile-related arteries is only one of them. Close collaboration with a urologist and exclusion of other aetiologies of ED prior to angiography is critical in patient selection and successful outcome.

\section{Author contributions}

As an editor of the journal, BP Yan was not involved in the peer review process of the article. BP Yan contributed to performance of the procedure, drafting of the article, and critical revision of the content.

\section{Declaration}

The author has disclosed no conflicts of interest. The author 
had full access to the data, contributed to the study, approved the final version for publication, and takes responsibility for its accuracy and integrity.

\section{References}

1. NIH Consensus Conference. Impotence. NIH Consensus Development Panel on Impotence. JAMA 1993;270:83-90.

2. Hatzimouratidis K, Hatzichristou DG. A comparative review of the options for treatment of erectile dysfunction: which treatment for which patient? Drugs 2005;65:162150.

3. Philip F, Shishehbor MH, Desai MY, Schoenhagen P, Ellis S, Kapadia SR. Characterization of internal pudendal artery atherosclerosis using aortography and multi-detector computed angiography. Catheter Cardiovasc Interv

\section{3;82:E516-21}

4. Zaki H, Nammas W, Shawky A, Mortada A, Zaki T. Prevalence of internal pudendal artery disease in diabetic patients with erectile dysfunction and angiographically documented multi-vessel coronary artery disease. Egypt Heart J 2013;65:87-91.

5. Rogers JH, Goldstein I, Kandzari DE, et al. Zotarolimuseluting peripheral stents for the treatment of erectile dysfunction in subjects with suboptimal response to phosphodiesterase-5 inhibitors. J Am Coll Cardiol 2012;60:2618-27.

6. Wang TD, Lee WJ, Yang SC, et al. Safety and six-month durability of angioplasty for isolated penile artery stenoses in patients with erectile dysfunction: a first-in-man study. EuroIntervention 2014;10:147-56. 